\author{
Unsere Ziele sind: \\ _Qualitätsindikatoren in der ambu- \\ lanten Palliativmedizin zu definieren \\ und weiterzuentwickeln, \\ _Betroffene und Angehörige über die \\ Möglichkeiten einer fachgerechten \\ palliativmedizinischen Versorgung zu \\ informieren, \\ _die Diskussion über ethische und \\ rechtliche Fragestellungen am \\ Lebensende anzustoßen und zu \\ vertiefen, \\ _ein langfristiger Kulturwandel im \\ Umgang mit Tod und Sterben.
}

\section{Berufsverband der Palliativmediziner in Westfalen-Lippe e.V.}

Geschäftsstelle:

Sabine Schäfer

Dieckmannstrasse 200

48161 Münster

Tel. 0251 / 5308-9960

info@bv-palliativmediziner.de

\author{
Öffentlichkeitsarbeit: \\ Dr. med. Eberhard A. Lux \\ Klinik für Schmerz- und \\ Palliativmedizin am \\ Klinikum St.-Marien-Hospital Lünen \\ Tel. 02306 / 77-2920 \\ Fax. $02306 / 77-2921$ \\ drlux@web.de
}

www.bv-palliativmediziner.de

\title{
Berufsverband gegründet
}

\section{Mit Engagement für die Palliativmedizin!}

W ie viele Jahre haben wir uns eine im ambulanten wie stationären Bereich bessere Versorgung unserer Patienten am Lebensende gewünscht - und über wie viele Jahre sind wir von einer Ankündigung zur nächsten zwischen Euphorie und Enttäuschung geschwankt. Mit der Implementierung der „Vereinbarung zur Umsetzung der ambulanten palliativmedizinischen Versorgung von unheilbar erkrankten Patienten im häuslichen Umfeld" haben sich die ambulanten Strukturen in Westfalen-Lippe in den letzten Jahren deutlich verändert. Unter Führung ambulant tätiger Palliativmediziner, in Kooperation mit Haus- und Fachärzten, aber auch mit Krankenhäusern, Altenheimen und stationären Hospizen sowie ambulanten Hospizvereinen haben wir begonnen, eine bedarfsgerechte Versorgung von Patienten am Lebensende zu gestalten und haben bereits deutliche Versorgungsengpässe überwunden.

Immerhin verstarben 2010 in Westfalen-Lippe $68,7 \%$ der 5.277 von uns versorgten Patienten zu Hause, 1,2\% auf Palliativstationen und $15,4 \%$ in stationären Hospizen - allerdings regional noch mit großem Unterschied. Wir erreichen mit unserer koordinierten Arbeit immer noch zu wenige Menschen und noch zu häufig wird der Notarzt zum sterbenden Patienten gerufen. Aber die Bereitschaft der Kostenträger, die geschaffene Organisationsstruktur zu erhalten und zu optimieren, interpretieren wir als Hinweis dafür, dass unser engagiertes Tun nicht nur den Bedürfnissen unserer Patienten und deren Angehörigen entspricht, sondern auch unnötige Kosten im Gesundheitssystem einspart.

Mit der Gründung unseres Berufsverbandes - Berufsverband der Palliativmediziner Westfalen-Lippe e.V. - festigen wir tragfähige Palliative-Care-Strukturen und Versorgungsangebote für unsere $\mathrm{Pa}$ tienten. Wir vertreten damit unsere gebündelte Kompetenz nach außen - vor allen Dingen gegenüber unseren Kostenträgern und der Politik. Wir kämpfen für eine solide finanzielle Basis unserer Strukturen und für die Weiterentwicklung der palliativmedizinischen Versorgung - in enger Kooperation mit allen im palliativen Netzwerk vertretenen Personen und Organisationen.

Unser mehrjähriges erfolgreiches Handeln hat gezeigt, dass fließende Übergänge von allgemeiner und spezialisierter ambulanter Palliativversorgung der Notwendigkeit einer Palliativversorgung in der Breite gerecht werden. Und mit der Einführung eines Operationen- und Prozedurenschlüssels (OPS) für spezialisierte stationäre Palliativmedizin wird sich ab 2014 in gleicher Weise in Kliniken eine bedarfsgerechte, palliativmedizinische Versorgungslandschaft etablieren. Auch unsere praktischen Erfahrungen und Ergebnisse bestätigen, dass der eingeschlagene Weg richtig ist, dass aber eine adäquate Begleitforschung die Grundlage weiterer Verbesserungen sein muss. Daran arbeiten wir ebenso.

Es ist uns ein Anliegen, allen Mitgliedern beratend und unterstützend zur Seite zu stehen, unabhängig davon, ob sie ambulant oder klinisch tätig sind. Und zwar in jeder Hinsicht: im Hinblick auf medizinisch fachliche Fragen, unter anderem durch entsprechende Fort-und Weiterbildungsangebote, auf medizinisch-ethische Fragen oder auf medizinisch-rechtliche Fragen, beispielsweise durch Benennung von geeigneten und befähigten Gutachtern gegenüber Schlichtungsstellen und Gerichten. Die Zeitschrift „Angewandte Schmerztherapie und Palliativmedizin“ bietet uns dafür eine Kommunikationsplattform.

Als Verantwortlicher für die Öffentlichkeitsarbeit unseres Berufsverbandes wünsche ich uns ein erfolgreiches und kollegiales Miteinander zum Wohl unserer Patienten.

Dr. Eberhard A. Lux, Lünen 